\title{
Synthesis and Antiacetylcholinesterase Activity of New D-Glyceraldehyde Heterocyclic Derivatives
}

\author{
Cecilia M. Scorzo, ${ }^{a}$ Mirta L. Fascio,${ }^{a}$ Norma B. D’Accorso, ${ }^{*}, a$ \\ Margarita Gutiérrez Cabrera ${ }^{b}$ and Luís Astudillo Saavedra ${ }^{b}$
}

\author{
${ }^{a}$ CIHIDECAR-CONICET. Departamento de Química Orgánica, Facultad de Ciencias Exactas y Naturales, \\ Universidad de Buenos Aires, Ciudad Universitaria, Pabellón II, 1428 Buenos Aires, Argentina \\ ${ }^{b}$ Laboratorio de Síntesis Orgánica, Instituto de Química de Productos Naturales, \\ Universidad de Talca, Casilla 747, Talca, Chile
}

\begin{abstract}
Neste trabalho são apresentados os procedimentos para a síntese de diferentes compostos heterocíclicos a partir do 2,3-O-isopropilideno-D-gliceraldeído: ciclização intramolecular, cicloadição 1,3-dipolar e reação de acoplamento bimolecular. Os produtos sintetizados foram caracterizados por espectroscopia de ${ }^{1} \mathrm{H}$ RMN e ${ }^{13} \mathrm{C}$ RMN e análise elementar. Os novos heterocíclicos e seus derivados (12 compostos) foram testados como inhibidores da enzima acetilcolinesterase.
\end{abstract}

We report herein the convenient procedures for the syntheses of different heterocyclic compounds from 2,3-O-isopropylidene-D-glyceraldehyde using intramolecular cyclization, 1,3-dipolar cycloaddition or bimolecular coupling reactions. The products were characterized by ${ }^{1} \mathrm{H}$ and ${ }^{13} \mathrm{C}$ NMR spectroscopy and elemental analysis. The new heterocycles and their derivatives were evaluated as inhibitors of acetylcholinesterase enzyme.

Keywords: D-glyceraldehyde, heterocyclic compounds, cycloaddition, acetylcholinesterase, Alzheimer's disease

\section{Introduction}

Alzheimer's disease $(\mathrm{AD})$ is a progressive, degenerative, and irreversible disorder that causes intellectual impairment and cognitive dysfunction. ${ }^{1}$ In the last decade, treatment for AD has been based on the "cholinergic hypothesis". ${ }^{2}$ This hypothesis suggested that patients with AD suffer from a deficit of cholinergic function in the brain such as decrease in hipocampal and cortical levels of acetylcholine (ACh) and associated enzyme choline transferase. Inhibition of acetylcholinesterase (AChE), an enzyme responsible for the metabolic break-down of $\mathrm{ACh}$, can restore the level of ACh in the brain. ${ }^{3}$ Hence the development of drugs for more serious AD is essential.

In the search for new molecules with the potential effect of inhibiting the enzyme acetylcholinesterase it is important to note that many heterocycles have shown

*e-mail: norma@qo.fcen.uba.ar significant inhibitory activity on this enzyme, including three of the four AD drugs currently on the market. ${ }^{4}$

The series of heterocycles known as benzoisoxazoles have been developed as potent and selective inhibitors of the enzime $\mathrm{AChE}$ with $\mathrm{IC}_{50}$ values between 0.8-14 nM. ${ }^{5}$ In addition, ester derivatives of tetrahydroazocines were synthesized and evaluated as inhibitors of the enzyme AChE, showing to be selective to the enzyme with $\mathrm{IC}_{50}$ values in the range of 5-40 $\mu \mathrm{M} .{ }^{6}$ A series of tacrine analogues has also been synthesized and evaluated as inhibitors of acetylcholinesterase/butyrylcholinesterase and modulators of $\mathrm{Ca}^{+2}$ channels and nicotinic receptors. $^{7}$

In the present study, a series of new D-glyceraldehyde heterocyclic derivatives were synthesized and were evaluated as potential inhibitors of acetylcholinesterase, so that current approaches to the treatment of cognitive and behavioral symptoms of AD make use of structurally diverse cholinesterase inhibitors. 


\section{Results and Discussion}

The heterocyclic compounds and their derivatives were synthesized from 2,3-O-isopropylidene-D-glyceraldehyde using simple and efficient methodologies (Scheme 1).

Compound 3 was obtained using intramolecular heterocyclization as we have previously described. ${ }^{8}$ In turn compounds $\mathbf{5}, \mathbf{6}$ and $\mathbf{8}$ were synthesized by 1,3-dipolar cycloaddition from the 2,3-O-isopropylideneD-glyceraldehyde oxime using alkynes ${ }^{9}$ or alkenes ${ }^{10}$ as dipolarophiles. The 1,2,4-oxadiazole derivatives (11 and 12) were obtained by cyclization of $O$-acyl amidoxime, which were synthesized using the technique described in the literature. ${ }^{11}$ Compounds 13-17 were obtained by deprotection of the corresponding precursors with acid medium. After purification by chromatographic column or recrystallization, compounds 2, 5-7, 11-14, 16 and 17 were obtained with moderate yields (see experimental) and were characterized by NMR spectroscopy. Spectroscopic data were in full agreement with those expected.

The syntheses of $\mathbf{3}, \mathbf{8}$ and $\mathbf{1 5}$ involved the formation of new asymmetric centre. Spectroscopic analysis of these compounds showed both diasteromers, in quasiequimolecular ratio. These facts can be explained because during the process of cyclization, intramolecular heterocyclization (compound 3 ) or 1,3-dipolar cycloaddition (compounds 8 and 15), there is no any chiral induction.

The compounds 3, 5-8 and 11-17 (Scheme1) were evaluated as potential inhibitors of the enzyme $\mathrm{AChE}$. AChE inhibitory activity was measured at room temperature by the colorimetric method previously described. ${ }^{12}$ The results of enzymatic inhibition are shown in Table 1 expressly as $\mathrm{IC}_{50}$ in $\mu \mathrm{g} \mathrm{mL}^{-1}$ and $\mu \mathrm{M}$.

All compounds showed moderate inhibitory activity of AChE, being compounds $\mathbf{3 , 5}$ and $\mathbf{7}$ with $\mathrm{IC}_{50}$ values of 110 , 220 and $190 \mu \mathrm{M}$ respectively. The most active compounds possess one of the following structural characteristics: a dioxolanic ring $(\mathbf{3}, \mathbf{5}$ and $\mathbf{7})$, an acetate group (3), a larger number of carbon atoms $(\mathbf{5}, \mathbf{7})$.

\section{Conclusion}

In summary, a new group of heterocyclic derivatives of 2,3-O-isopropylidene-D-glyceraldehyde with protected and unprotected hydroxyl groups were efficiently synthesized. The new derivatives were characterized by ${ }^{1} \mathrm{HNMR},{ }^{13} \mathrm{C}$ NMR and 2D heteronuclear techniques. Twelve compounds were evaluated as inhibitors of acetylcholinesterase showing moderate to low activity. The most active compounds were $\mathbf{3 , 5}$ and $\mathbf{7}$ with $\mathrm{IC}_{50}$ less than $220 \mu \mathrm{M}$.

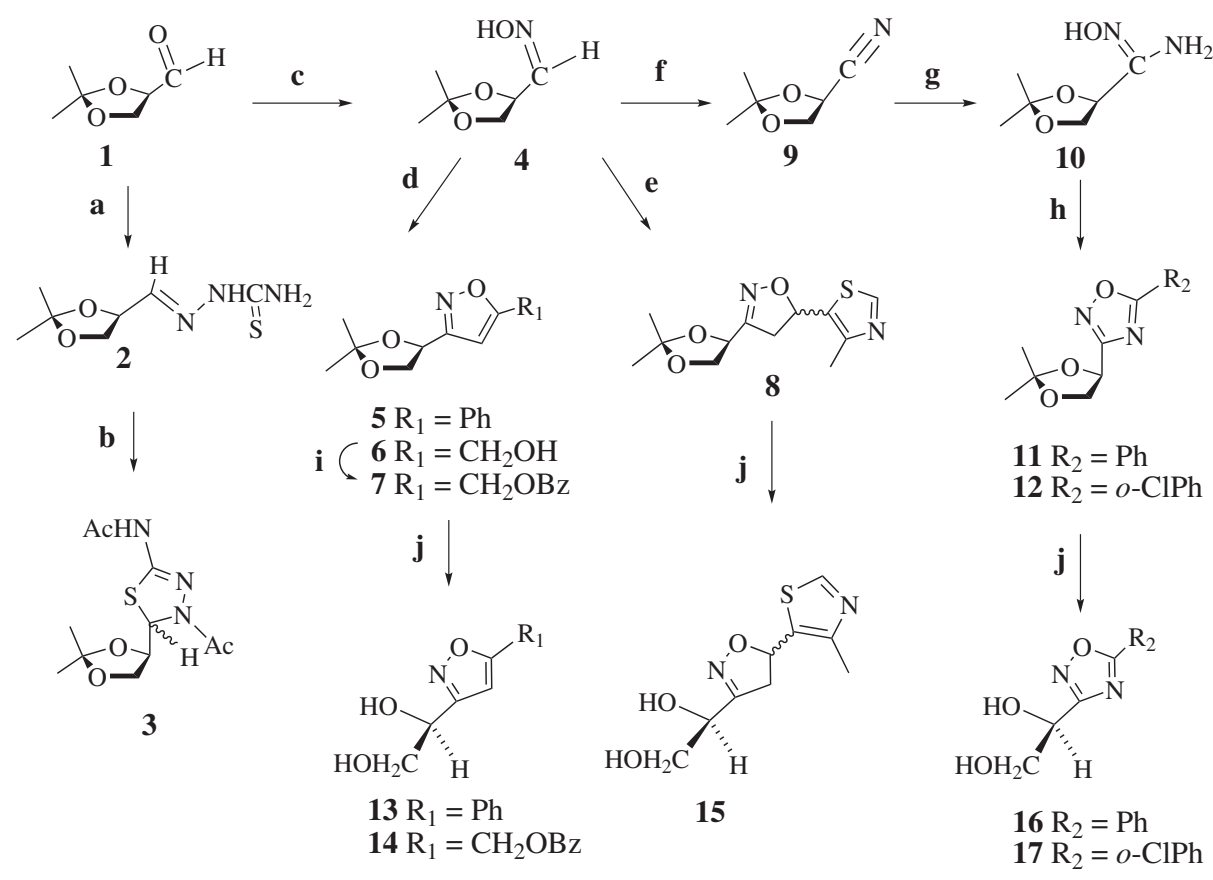

a) $\mathrm{NH}_{2} \mathrm{CSNHNH}_{2}, \mathrm{EtOH}$; b) $\left(\mathrm{CH}_{3} \mathrm{CO}\right)_{2} \mathrm{O}, \mathrm{Py}$; c) $\mathrm{NH}_{2} \mathrm{OH} . \mathrm{HCl}, \mathrm{Na}_{2} \mathrm{CO}_{3}, \mathrm{H}_{2} \mathrm{O}$; d) Chloramine-T,

phenylacetylene, EtOH- $\mathrm{H}_{2} \mathrm{O}$ (5); chloramine-T, propargyl alcohol (6); e) 4-methyl-5-vinylthiazole, chloramine-

T, EtOH-H $\mathrm{H}_{2} \mathrm{O}$; f) $\mathrm{SOCl}_{2}, \mathrm{Na}_{2} \mathrm{CO}_{3}, \mathrm{CH}_{2} \mathrm{Cl}_{2}$ (anhydrous); g) $\mathrm{NH}_{2} \mathrm{OH} . \mathrm{HCl}, \mathrm{Na}_{2} \mathrm{CO}_{3}, \mathrm{MeOH}-\mathrm{H}_{2} \mathrm{O}$; h) $\mathrm{PhCO}_{2} \mathrm{H}$

(11) or $o-\mathrm{Cl}-\mathrm{PhCO}_{2} \mathrm{H}(\mathbf{1 2}), \mathrm{DCC}, \mathrm{CH}_{2} \mathrm{Cl}_{2}$ (anhydrous), $3 \mathrm{~h}$ at rt, then $110-120^{\circ} \mathrm{C}$; i) $\mathrm{PhCOCl}, \mathrm{Py}$; j) TFA, $\mathrm{H}_{2} \mathrm{O}$. 
Table 1. $\mathrm{IC}_{50}$ for $\mathrm{AChE}$ inhibition for some selected compounds

\begin{tabular}{lcc}
\hline Compound & $\mu \mathrm{g} \mathrm{mL}^{-1}$ & $\mu \mathrm{M}$ \\
\hline $\mathbf{3}$ & $\mathbf{3 1}$ & 110 \\
$\mathbf{5}$ & $\mathbf{5 4}$ & 220 \\
$\mathbf{6}$ & 272 & 1370 \\
$\mathbf{7}$ & $\mathbf{5 6}$ & 190 \\
$\mathbf{8}$ & 196 & 730 \\
$\mathbf{1 1}$ & 127 & 520 \\
$\mathbf{1 2}$ & 191 & 680 \\
$\mathbf{1 3}$ & 230 & 1120 \\
$\mathbf{1 4}$ & $>500$ & - \\
$\mathbf{1 5}$ & 114 & 500 \\
$\mathbf{1 6}$ & $>500$ & - \\
$\mathbf{1 7}$ & 178 & 740 \\
Galanthamine & 1.1 & 3.0 \\
\hline
\end{tabular}

\section{Experimental}

Optical rotations were recorded at $20{ }^{\circ} \mathrm{C}$ on a Perkin Elmer 343 polarimeter, and the melting points were uncorrected. ${ }^{1} \mathrm{H}$ NMR and ${ }^{13} \mathrm{C}$ NMR spectra were recorded on a Bruker AC-200 spectrometer at $200 \mathrm{MHz}$ and $50 \mathrm{MHz}$. Elemental analyses were performed by UMYMFOR, CONICET-University of Buenos Aires, Argentina. Analytical TLC was conducted on Silica Gel 60G (Merck) on precoated plates and visualization was made by UV light and ethanol/sulfuric acid (10:1) or cerium molibdate, followed by heating. Column-chromatographic separations were performed on Silica Gel (240-400 mesh, Merck). Solvents were reagent grade and, in most cases, dried and distilled before use according to standard procedures.

\section{2,3-O-Isopropylidene-D-glycerothiosemicarbazone (2)}

The 2,3-O-isopropylidene-D-glyceraldehyde ${ }^{13}(0.98 \mathrm{~g}$, $7.50 \mathrm{mmol}$ ) was dissolved in ethanol and heated with a small excess of thiosemicarbazide. The reaction was monitored by TLC until no starting material was observed. The reaction medium was evaporated and the residue purified by flash chromatography (alumina, cyclohexane/ethyl acetate, 7:3). Compound 2 was obtained as yellow syrup (1.20 g, 78.3\%). ${ }^{1} \mathrm{H}$ NMR (200 MHz, $\mathrm{CDCl}_{3}$ ): $\delta 1.33(\mathrm{~s}, 3 \mathrm{H}), 1.37(\mathrm{~s}, 3 \mathrm{H})$ (methyl protons); $3.88(\mathrm{dd}, 1 \mathrm{H}), 4.12(\mathrm{dd}, 1 \mathrm{H}), 4.57$ (dd, 1H) (carbohydrate protons); $7.10(\mathrm{~s}, 1 \mathrm{H}), 7.30$ (s, 1H), 7.33 (s, $1 \mathrm{H})$ (thiourea protons); $10.65(\mathrm{~s}, 1 \mathrm{H})(\mathrm{CH}=\mathrm{N}) .{ }^{13} \mathrm{C} \mathrm{NMR}(50$ $\mathrm{MHz}, \mathrm{CDCl}_{3}$ ): $\delta 25.25,26.40$ (methyl carbons); 67.05, 74.79 (carbohydrate carbons); 110.26 (isopropylidene carbon); 144.56 (imine carbon), 178.19 (thiocarbonyl carbon). Elemental Anal. Calc. for $\mathrm{C}_{7} \mathrm{H}_{13} \mathrm{~N}_{3} \mathrm{O}_{2} \mathrm{~S}$ : C, 41.36; H, 6.45; N, 20.67. Found: C, 41.35; H, 6.54; N, 20.41.
2-Acetamido-4-N-acetyl-5-[(4R)-2,2-dimethyl-1,3dioxolan-4-yl]-1,3,4-thiadiazoline (3)

Thiosemicarbazone $2(0.19 \mathrm{~g}, 0.95 \mathrm{mmol})$, acetic anhydride $(3 \mathrm{~mL})$ and pyridine $(3 \mathrm{~mL})$ were heated at reflux and the reaction was monitored by TLC until the starting material disappeared. The reaction was left at room temperature and then stopped by adding some water. The reaction mixture was extracted with chloroform and the organic phase was dried, evaporated and the residue purified using flash chromatography (silica gel, cyclohexane/ acetone, 8:2). After purification, a diasteromeric mixture (54:46 ratio), of thiadiazoline 3 (91.3 $\mathrm{mg}, 33.0 \%)$ was obtained as a amorphous white solid. ${ }^{1} \mathrm{H}$ NMR $(200 \mathrm{MHz}$, $\left.\mathrm{CDCl}_{3}\right): \delta 1.32(\mathrm{~s}, 3 \mathrm{H}), 1.33(\mathrm{~s}, 3 \mathrm{H}), 1.44(\mathrm{~s}, 3 \mathrm{H}), 1.46$ (s, 3H), 2.21 (s, 3H), $2.22(\mathrm{~s}, 3 \mathrm{H}), 2.23(\mathrm{~s}, 3 \mathrm{H}), 2.24(\mathrm{~s}$, $3 \mathrm{H})$ (methyl protons); 3.85-4.10 (m, $2 \mathrm{H}), 3.90(\mathrm{~m}, 1 \mathrm{H})$, $4.04(\mathrm{~m}, 1 \mathrm{H}), 4.37(\mathrm{~m}, 1 \mathrm{H}), 4.51(\mathrm{~m}, 1 \mathrm{H})$ (carbohydrate protons); $5.95(\mathrm{~d}, 1 \mathrm{H}), 6.13(\mathrm{~d}, 1 \mathrm{H})$ (thiadiazolyl protons); $10.00(\mathrm{~s}, 1 \mathrm{H}), 10.03(\mathrm{~s}, 1 \mathrm{H})$ (amide proton). ${ }^{13} \mathrm{C}$ NMR (50 $\left.\mathrm{MHz}, \mathrm{CDCl}_{3}\right): \delta 21.74,21.77,22.97,23.02,24.83,25.17$, 26.01, 26.13 (methyl carbons); 65.02, 66.62, 66.37, 66.97 (carbohydrate carbons); 110.07, 110.29 (isopropylidene carbon); 75.27, 76.47, 148.23, 148.27 (thiadiazolyl carbons); 169.08, 169.22, 169.82, 169.96 (carbonyl carbons). Elemental Anal. Calc. for $\mathrm{C}_{11} \mathrm{H}_{17} \mathrm{~N}_{3} \mathrm{O}_{4} \mathrm{~S}: \mathrm{C}, 45.98$; H, 5.96. Found: C, 45.68; H, 6.08 .

General procedure 1: synthesis of isoxazol and isoxazoline derivatives $(\mathbf{5}, \mathbf{6}, \mathbf{8})$

An ethanolic solution of 2,3-O-isopropylidene-Dglyceraldehydeoxime ${ }^{14}(4)(1.00 \mathrm{~g}, 6.90 \mathrm{mmol})$ was added slowly, at room temperature, to an ethanolic solution of dipolarophiles with an excess of chloramine-T. The reaction was monitored by TLC (cyclohexane/acetone, 7:3). The product was purified by column chromatography (silica gel, cyclohexane/acetone, with different ratio). The respectively compounds were characterized as follows.

\section{3-[(4S)-2,2-Dimethyl-1,3-dioxolan-4-yl]-5-phenyl- isoxazole (5)}

General procedure 1 was applied using phenylacetylene $(1.30 \mathrm{~mL})$ as the dipolarophile yielding compound $\mathbf{5}$ $(0.56 \mathrm{~g}, 33 \%)$ as a syrup, $[\alpha]_{\mathrm{D}}+9.7\left(c 1, \mathrm{CHCl}_{3}\right) .{ }^{1} \mathrm{H} \mathrm{NMR}$ $\left(\mathrm{CDCl}_{3}\right): \delta 1.47$ (s, 3H), 1.53 (s, 3H) (methyl protons); $4.09(\mathrm{dd}, 1 \mathrm{H}), 4.39(\mathrm{dd}, 1 \mathrm{H}), 5.25(\mathrm{t}, 1 \mathrm{H})$ (carbohydrate protons); 6.59 (s, 1H) (isoxazolyl proton); 7.33-7.90 (m, $5 \mathrm{H})$ (aromatic protons). ${ }^{13} \mathrm{C} \mathrm{NMR}\left(\mathrm{CDCl}_{3}\right): \delta 25.38$, 26.38 (methyl carbons); 68.94, 70.36 (carbohydrate carbons); 110.34 (isopropylidene carbon); 125.72, 127.14, 128.88, 130.19 (aromatic carbons); 97.60, 164.24, 170.26 
(isoxazolyl carbons). Elemental Anal. Calc. for $\mathrm{C}_{14} \mathrm{H}_{15} \mathrm{NO}_{3}$ : C, 68.57; H, 6.12. Found: C, 68.30; H, 6.22.

\section{5-Hydroxymethyl-3-[(4S)-2,2-dimethyl-1,3-dioxolan-4-yl] isoxazole (6)}

General procedure 1 was applied to 2-propyn-1-ol $(5.1 \mathrm{~mL})$ as the dipolarophile yielding compound 6 $(0.81 \mathrm{~g}, 59 \%)$ as a syrup which was purified by column chromatography (silica gel, toluene/ethyl acetate, 8:2). $[\alpha]_{\mathrm{D}}+16.8\left(c 1, \mathrm{CHCl}_{3}\right) .{ }^{1} \mathrm{H} \mathrm{NMR}\left(\mathrm{CDCl}_{3}\right): \delta 1.38(\mathrm{~s}$, $3 \mathrm{H}), 1.41$ (s, 3H) (methyl protons); 3.93 (dd, 1H), 4.27 $(\mathrm{dd}, 1 \mathrm{H}), 5.11(\mathrm{t}, 1 \mathrm{H})$ (carbohydrate protons); $4.64(\mathrm{~s}, 2 \mathrm{H})$ (methylenic protons); 6.25 (s, 1H) (isoxazolyl proton). ${ }^{13} \mathrm{C}$ NMR $\left(\mathrm{CDCl}_{3}\right): \delta 25.18,26.17$ (methyl carbons); 55.89 (methylenic carbon); 68.73, 70.04 (carbohydrate carbons); 110.39 (isopropylidene carbon); 99.99, 163.55, 172.19 (isoxazolyl carbons). Elemental Anal. Calc. for $\mathrm{C}_{9} \mathrm{H}_{13} \mathrm{NO}_{4}$ : C, 54.26; H, 6.58; N, 7.03. Found: C, 54.04; $\mathrm{H}, 6.57 ; \mathrm{N}, 7.37$.

\section{5-Benzoyloxymethyl-3-[(4S)-2,2-dimethyl-1,3-dioxolan- 4-yl]isoxazole (7)}

The 5-hydroxymethyl-3-[(4S)-2,2-dimethyl-1,3dioxolan-4-yl)isoxazole (6) $(0.42 \mathrm{~g}, 2.09 \mathrm{mmol})$ was suspended in $2 \mathrm{~mL}$ of pyridine and benzoyl chloride $(0.3 \mathrm{~mL}$, $2.58 \mathrm{mmol}$ ) was added. The reaction was monitored by TLC until no starting material was observed. The reaction medium was evaporated and the residue purified by flash chromatography (silica gel, cyclohexane/acetone, 8:2). Compound $7(0.51 \mathrm{~g}, 81 \%)$ was obtained as a syrup, $[\alpha]_{\mathrm{D}}+11.7\left(c 1, \mathrm{CHCl}_{3}\right) .{ }^{1} \mathrm{H} \mathrm{NMR}\left(\mathrm{CDCl}_{3}\right): \delta 1.45(\mathrm{~s}, 3 \mathrm{H})$, 1.49 (s, 3H) (methyl protons); $4.04(\mathrm{dd}, 1 \mathrm{H}), 4.36(\mathrm{dd}$, $1 \mathrm{H}), 5.22(\mathrm{dd}, 1 \mathrm{H})$ (carbohydrate protons); $5.41(\mathrm{~s}, 2 \mathrm{H})$ (methylenic protons); 6.46 (s, 1H) (isoxazolyl proton); 7.40-8.11 (m, 5H) (aromatic protons). ${ }^{13} \mathrm{C} \mathrm{NMR}\left(\mathrm{CDCl}_{3}\right): \delta$ 25.34, 26.36 (methyl carbons); 56.64 (methylenic carbon); 68.86, 70.18 (carbohydrate carbons); 110.43 (isopropylidene carbon); 102.43, 163.85, 167.16 (isoxazolyl carbons); 128.44, 128.98, 129.78, 133.47 (aromatic carbons); 165.65 (carbonyl carbon). Elemental Anal. Calc. for $\mathrm{C}_{16} \mathrm{H}_{17} \mathrm{NO}_{5}$ : C, 63.36; H, 5.65. Found: C, 63.19; H, 5.99.

\section{3-[(4S)-2,2-Dimethyl-1,3-dioxolan-4-yl]-5-(4- methylthiazolyl)isoxazoline (8)}

General procedure 1 was applied using 4-methyl-5vinylthiazole $(1.6 \mathrm{~mL})$ as the dipolarophile yielding a diastereomeric mixture (56:44 ratio) of $\mathbf{8}(0.94 \mathrm{~g}, 51 \%)$ as a syrup, which was purified by column chromatography (silica gel, mixture cyclohexane/acetone, starting from 9:1). ${ }^{1} \mathrm{H}$ NMR $\left(\mathrm{CDCl}_{3}\right): \delta 1.41$ (s, 3H), $1.46(\mathrm{~s}, 3 \mathrm{H}), 2.42(\mathrm{~s}, 3 \mathrm{H})$ (methyl protons); 4.04 (dd, 1H), $4.06(\mathrm{dd}, 1 \mathrm{H}), 4.26(\mathrm{dd}$,
1H), $4.27(\mathrm{dd}, 1 \mathrm{H}), 4.98(\mathrm{~m}, 1 \mathrm{H}), 5.00(\mathrm{~m}, 1 \mathrm{H})$ (carbohydrate protons); $3.02(\mathrm{dd}, 1 \mathrm{H}), 3.07(\mathrm{dd}, 1 \mathrm{H}), 3.52(\mathrm{dd}, 1 \mathrm{H})$, 3.57 (dd, 1H), 5.87 (dd, 1H), 5.90 (dd, 1H) (isoxazolidyl protons); $8.68(\mathrm{~s}, 1 \mathrm{H}), 8.67$ (s, 1H) (thiazolyl protons). ${ }^{13} \mathrm{C}$ NMR $\left(\mathrm{CDCl}_{3}\right): \delta 14.90,15.19,24.88,24.90,26.09$ (methyl carbons); 66.88, 66.97, 70.78, 70.82 (carbohydrate carbons); 110.36, 110.38 (isopropylidene carbons); 41.86, 41.89, 75.35, 75.56, 150.38, 150.42 (isoxazolidyl carbons); 129.43, 130.75, 151.13, 151.32, 157.88, 157.99 (thiazolyl carbons). Elemental Anal. Calc. for $\mathrm{C}_{12} \mathrm{H}_{16} \mathrm{~N}_{2} \mathrm{O}_{3} \mathrm{~S}$ : C, 53.71; H, 6.01. Found: C, 53.64; H, 5.78.

\section{2,3-O-Isopropylidene-D-glyceronitrile (9)}

Anhydrous $\mathrm{CH}_{2} \mathrm{Cl}_{2}(20 \mathrm{~mL})$ was added to a mixture of $4^{14}(1.23 \mathrm{~g}, 8.5 \mathrm{mmol})$ and $\mathrm{Na}_{2} \mathrm{CO}_{3}(3.87 \mathrm{~g}, 36.5 \mathrm{mmol})$ in $\mathrm{Ar}$ atmosphere. The reaction mixture was cooled at $0{ }^{\circ} \mathrm{C}$ and $1 \mathrm{~mL}$ of $\mathrm{SOCl}_{2}$ was added slowly. The reaction was monitored by TLC until the starting material disappeared. The reaction was poured into finely crushed ice and the product was extracted with $\mathrm{CH}_{2} \mathrm{Cl}_{2}$. The solution was dried with anhydrous $\mathrm{Na}_{2} \mathrm{SO}_{4}$, filtered and evaporated. Compound 9 was obtained as a syrup $(0.73 \mathrm{~g}, 68 \%),[\alpha]_{\mathrm{D}}+9.3(c 1$, $\left.\mathrm{CHCl}_{3}\right) .{ }^{1} \mathrm{H} \mathrm{NMR}\left(\mathrm{CDCl}_{3}\right): \delta 1.40(\mathrm{~s}, 3 \mathrm{H}), 1.55(\mathrm{~s}, 3 \mathrm{H})$ (methyl protons); 4.19 (dd, 1H), 4.29 (dd, 1H), 4.77 (dd, $1 \mathrm{H})$ (carbohydrate protons). ${ }^{13} \mathrm{C} \mathrm{NMR}\left(\mathrm{CDCl}_{3}\right): \delta 25.41$, 25.66 (methyl carbons); 63.11, 67.97 (carbohydrate carbons); 112.66 (isopropylidene carbon); 118.21 (cyano carbon).

\section{2,3-O-Isopropylidene-D-glyceroamidoxime (10)}

A methanolic solution of compound $9(0.72 \mathrm{~g}$, $5.65 \mathrm{mmol}$ ) and a aqueous solution of hydroxylamine [prepared from hydroxylamine hydrochloride $(0.77 \mathrm{~g}$, $11.1 \mathrm{mmol})$ and sodium carbonate $(0.59 \mathrm{~g}, 5.6 \mathrm{mmol})$ in $5 \mathrm{~mL}$ of water] was heated at $60{ }^{\circ} \mathrm{C}$; the reaction mixture was monitored by TLC until the starting material disappeared. The reaction medium was evaporated and the residue purified by flash chromatography (silica gel, cyclohexane/acetone, 8:2). Compound $\mathbf{1 0}(0.51 \mathrm{~g}, 57 \%)$ was obtained as a syrup. ${ }^{1} \mathrm{H}$ NMR $\left(\mathrm{CDCl}_{3}\right): \delta 1.39(\mathrm{~s}, 3 \mathrm{H})$, 1.49 (s, 3H) (methyl protons); 3.99 (dd, 1H), 4.18 (dd, $1 \mathrm{H}), 4.55(\mathrm{dd}, 1 \mathrm{H})$ (carbohydrate protons); 4.84 (br. s, 2H) (amine protons); $8.30(\mathrm{~s}, 1 \mathrm{H})$ (hydroxyl proton). ${ }^{13} \mathrm{C}$ NMR $\left(\mathrm{CDCl}_{3}\right): \delta$ 25.02, 26.17 (methyl carbons); 67.95, 72.88 (carbohydrate carbons); 110.22 (isopropylidene carbon); 152.93 (amidoxime carbon). Elemental Anal. Calc. for $\mathrm{C}_{6} \mathrm{H}_{12} \mathrm{~N}_{2} \mathrm{O}_{3}: \mathrm{C}, 44.99 ; \mathrm{H}, 7.55$. Found: $\mathrm{C}, 45.02 ; \mathrm{H}, 7.99$.

5-Phenyl-3-[(4S)-2,2-dimethyl-1,3-dioxolan-4-yl]-1,2,4oxadiazole (11)

Benzoic acid $(0.38 \mathrm{~g}, 3.15 \mathrm{mmol})$ was added to a solution of compound $10(0.25 \mathrm{~g} ; 1.56 \mathrm{mmol})$ in anhydrous $\mathrm{CH}_{2} \mathrm{Cl}_{2}$ 
(15 mL), followed by DCC $(0.65 \mathrm{~g}, 3.15 \mathrm{mmol})$, and the reaction mixture was stirred at room temperature under a $\mathrm{N}_{2}$ atmosphere for $3 \mathrm{~h}$. The solid was filtered off, the solvent was evaporated and the residue was subjected to the cyclodehydration reaction at $110-120{ }^{\circ} \mathrm{C}$ for $4 \mathrm{~h}$. The product was purified by column chromatography (silica gel, cyclohexane/acetone, 9:1) yielding compound 11 (0.30 g, $78 \%)$ as a syrup, $[\alpha]_{\mathrm{D}}+16.7\left(c 1, \mathrm{CHCl}_{3}\right) .{ }^{1} \mathrm{H} \mathrm{NMR}\left(\mathrm{CDCl}_{3}\right)$ : $\delta 1.51(\mathrm{~s}, 3 \mathrm{H}), 1.59(\mathrm{~s}, 3 \mathrm{H})$ (methyl protons); $4.32(\mathrm{dd}, 1 \mathrm{H})$, $4.43(\mathrm{dd}, 1 \mathrm{H}), 5.30(\mathrm{t}, 1 \mathrm{H})$ (carbohydrate protons); 7.36-8.25 (m, 5H) (aromatic protons). ${ }^{13} \mathrm{C} \mathrm{NMR}\left(\mathrm{CDCl}_{3}\right): \delta 25.66$, 26.06 (methyl carbons); 67.89, 69.88 (carbohydrate carbons); 111.21 (isopropylidene carbon); 123.84, 128.11, 128.99, 132.85 (aromatic carbons); 169.78, 176.25 (oxadiazolyl carbons). Elemental Anal. Calc. for $\mathrm{C}_{13} \mathrm{H}_{14} \mathrm{~N}_{2} \mathrm{O}_{3}$ : C, 63.40; H, 5.73; N, 11.38. Found: C, 63.71; H, 5.87; N, 11.30.

\section{5-(2-Chlorophenyl)-3-[(4S)-2,2-dimethyl-1,3-dioxolan-4-} yl]-1,2,4-oxadiazole (12)

Applying the same procedure described for compound 11 but using $o$-chlorobenzoic acid $(0.49 \mathrm{~g}, 3.15 \mathrm{mmol})$, instead of benzoic acid, yielded compound $\mathbf{1 2}(0.17 \mathrm{~g}, 38 \%)$ as a syrup, $[\alpha]_{\mathrm{D}}+12.6\left(c 1, \mathrm{CHCl}_{3}\right) \cdot{ }^{1} \mathrm{H} \mathrm{NMR}\left(\mathrm{CDCl}_{3}\right)$ : $\delta 1.51(\mathrm{~s}, 3 \mathrm{H}), 1.60(\mathrm{~s}, 3 \mathrm{H})$ (methyl protons); 4.33 (dd, $1 \mathrm{H}), 4.43(\mathrm{dd}, 1 \mathrm{H}), 5.34(\mathrm{t}, 1 \mathrm{H})$ (carbohydrate protons); 7.32-8.19 (m, 4H) (phenyl protons). ${ }^{13} \mathrm{C}$ NMR $\left(\mathrm{CDCl}_{3}\right)$ : $\delta$ 25.76, 26.12 (methyl carbons); 68.07, 69.90 (carbohydrate carbons); 111.37 (isopropylidene carbon); 127.02, 128.76, 130.83, 131.42, 131.98, 133.25 (phenyl carbons); 169.66, 174.90 (oxadiazolyl carbons). Elemental Anal. Calc. for $\mathrm{C}_{13} \mathrm{H}_{13} \mathrm{~N}_{2} \mathrm{O}_{3} \mathrm{Cl}$ : C, 55.62; H, 4.67. Found: C, 55.45; H, 4.27.

\section{General procedure 2: deprotection of nucleoside analogues}

A mixture of $2.04 \mathrm{mmol}$ of 2,3-O-isopropylidene derivatives $(\mathbf{5}, \mathbf{7}, \mathbf{8}, \mathbf{1 1}, \mathbf{1 2}), 2 \mathrm{~mL}$ of TFA and $2 \mathrm{~mL}$ of water was stirred at room temperature for $4 \mathrm{~h}$. The reaction mixture was evaporated and the deprotected compounds were purified as described in each cases.

\section{3-[(1S)-Ethanediol-1-yl]-5-phenylisoxazole (13)}

Compound $\mathbf{1 3}$ was obtained as an amorphous solid recrystallized from toluene $\left(0.29 \mathrm{~g}, 70 \% ; \mathrm{mp} 88-89^{\circ} \mathrm{C}\right)$. $[\alpha]_{\mathrm{D}}+13.6\left(\mathrm{c} 1\right.$, methanol). ${ }^{1} \mathrm{H} \mathrm{NMR}\left(\left(\mathrm{CD}_{3}\right)_{2} \mathrm{CO}\right): \delta 3.67-$ $3.90(\mathrm{~m}, 2 \mathrm{H}), 4.86(\mathrm{dd}, 1 \mathrm{H})$ (carbohydrate protons); 6.89 (s, 1H) (isoxazolyl proton); 7.38-8.03 (m, 5H) (aromatic protons). ${ }^{13} \mathrm{C} \mathrm{NMR}\left(\mathrm{CD}_{3} \mathrm{OD}\right): \delta 66.50,68.54$ (carbohydrate carbons); 126.39, 128.49, 129.97, 130.94 (aromatic carbons); 99.34, 166.59, 169.96 (isoxazolyl carbons). Elemental Anal. Calc. for $\mathrm{C}_{11} \mathrm{H}_{11} \mathrm{NO}_{3}$ : C, 64.38; H, 5.40; N, 6.83. Found: C, 64.15; H, 5.25; N, 7.01.
5-Benzoyloxymethyl-3-[(1S)-ethanediol-1-yl]isoxazole (14)

Compound $\mathbf{1 4}$ was obtained as an amorphous solid recrystallized from water $\left(0.33 \mathrm{~g}, 78 \%\right.$; mp $\left.75-76{ }^{\circ} \mathrm{C}\right)$. $[\alpha]_{\mathrm{D}}+5.7$ (c 1, methanol). ${ }^{1} \mathrm{H}$ NMR $\left(\left(\mathrm{CD}_{3}\right)_{2} \mathrm{CO}\right): \delta 3.78$ $(\mathrm{d}, 2 \mathrm{H}), 4.86(\mathrm{t}, 1 \mathrm{H})$ (carbohydrate protons); $5.49(\mathrm{~s}, 2 \mathrm{H})$ (methylenic protons); $6.61(\mathrm{~s}, 1 \mathrm{H})$ (isoxazolyl proton); 7.32-8.15 (m, 5H) (aromatic protons). ${ }^{13} \mathrm{C} \mathrm{NMR}\left(\left(\mathrm{CD}_{3}\right)_{2} \mathrm{CO}\right)$ : $\delta 57.74$ (methylenic carbon); 66.21, 68.51 (carbohydrate carbons); 129.53, 130.22, 130.90, 134.53 (aromatic carbons); 103.78, 166.52, 168.27 (isoxazolyl carbons); 166.91 (carbonyl carbon). Elemental Anal. Calc. for $\mathrm{C}_{13} \mathrm{H}_{13} \mathrm{NO}_{5}$ : C, 59.31; H, 4.98; N, 5.32. Found: C, 58.94; H, 4.78; N, 5.35.

\section{3-[(1S)-Ethanediol-1-yl]-5-(4-methylthiazolyl)isoxazoline} (15)

A diastereomeric mixture (56:44 ratio) was obtained as an amorphous solid purified by silica gel flash chromatography using toluene:ethanol 8:2 as eluent (0.23 g, 54\%; mp 102-104 $\left.{ }^{\circ} \mathrm{C}\right) .{ }^{1} \mathrm{H}$ NMR (CD $\left.\mathrm{OD}\right): \delta$ $2.44(\mathrm{~s}, 6 \mathrm{H})$ (methyl protons); $3.72(\mathrm{~d}, 4 \mathrm{H}), 4.53(\mathrm{t}, 2 \mathrm{H})$ (carbohydrate protons); $3.07(\mathrm{ddd}, 1 \mathrm{H}), 3.08(\mathrm{ddd}, 1 \mathrm{H})$, 3.61 (ddd, 1H), 3.63 (ddd, 1H), 5.93 (dd, 2H) (isoxazolidyl protons); 8.86 (s, 2H) (thiadiazolyl protons). ${ }^{13} \mathrm{C}$ NMR (CD $\mathrm{OD}$ ): $\delta 14.94$ (methyl carbon); 65.04, 65.09, 69.17, 69.27 (carbohydrate carbons); 43.22, 43.41, 76.33, 150.98, 151.02 (isoxazolidyl carbons); 133.36, 133.48, 153.72, 161.66, 161.69 (thiadiazolyl carbons). Elemental Anal. Calc. for $\mathrm{C}_{9} \mathrm{H}_{12} \mathrm{~N}_{2} \mathrm{O}_{3} \mathrm{~S}$ : C, 47.35; H, 5.30. Found: C, 47.17; H, 5.09.

\section{5-Phenyl-3-[(1S)-ethanediol-1-yl]-1,2,4-oxadiazole (16)}

Compound 16 was obtained as an amorphous solid recrystallized from toluene $\left(0.11 \mathrm{~g}, 26.4 \%\right.$; $\left.\mathrm{mp} 84-85^{\circ} \mathrm{C}\right)$. $[\alpha]_{\mathrm{D}}+6.9\left(c 1\right.$, methanol). ${ }^{1} \mathrm{H}$ NMR $\left(\left(\mathrm{CD}_{3}\right)_{2} \mathrm{CO}\right): \delta 3.97(\mathrm{~d}$, 2H), $4.93(\mathrm{t}, 1 \mathrm{H})$ (carbohydrate protons); 7.49-8.33 (m, 5H) (aromatic protons). ${ }^{13} \mathrm{C}$ NMR $\left(\left(\mathrm{CD}_{3}\right)_{2} \mathrm{CO}\right): \delta 65.22,68.24$ (carbohydrate carbons); 125.00, 128.65, 130.15, 133.74 (aromatic carbons); 172.34, 176.22 (oxadiazolyl carbons). Elemental Anal. Calc. for $\mathrm{C}_{10} \mathrm{H}_{10} \mathrm{~N}_{2} \mathrm{O}_{3}: \mathrm{C}, 58.25 ; \mathrm{H}, 4.89$; N, 13.59. Found: C, 58.00; H, 4.76; N, 13.29 .

\section{5-(2-Chlorophenyl)-3-[(1S)-ethanediol-1-yl]-1,2,4- oxadiazole (17)}

Compound 17 was obtained as an amorphous solid recrystallized from toluene $\left(0.17 \mathrm{~g}, 39.5 \%\right.$; $\left.\mathrm{mp} 76-78^{\circ} \mathrm{C}\right)$. $[\alpha]_{\mathrm{D}}+5.9$ ( $c$ 1, methanol). ${ }^{1} \mathrm{H}$ NMR $\left(\mathrm{CD}_{3} \mathrm{OD}\right): \delta 3.85-3.95$ $(\mathrm{m}, 1 \mathrm{H}), 3.92-4.01(\mathrm{~m}, 1 \mathrm{H}), 4.93(\mathrm{t}, 1 \mathrm{H})$ (carbohydrate protons); 7.45-8.16 (m, $4 \mathrm{H}$ ) (aromatic protons). ${ }^{13} \mathrm{C}$ NMR (CD $\mathrm{OD}$ ): $\delta 66.13,69.32$ (carbohydrate carbons); 125.51, 129.43, 133.30, 133.98, 135.39, 135.64 (aromatic carbons); 173.23, 176.88 (oxadiazolyl carbons). Elemental Anal. 
Calc. for $\mathrm{C}_{10} \mathrm{H}_{9} \mathrm{~N}_{2} \mathrm{O}_{3} \mathrm{Cl}$ : C, 49.91; H, 3.77; N, 11.64. Found: C, 49.77; H, 3.64; N, 11.58

\section{Biological assay}

In the 96-well plates $50 \mu \mathrm{L}$ of the sample dissolved in phosphate buffer $\left(8 \mathrm{mmol} \mathrm{L}^{-1} \mathrm{~K}_{2} \mathrm{HPO}_{4}, 2.3 \mathrm{mmol} \mathrm{L}{ }^{-1}\right.$ $\mathrm{NaH}_{2} \mathrm{PO}_{4}, 150 \mathrm{mmol} \mathrm{L}-1 \mathrm{NaCl}$, and $0.05 \%$ Tween 20 at $\mathrm{pH} 7.6$ ) and $50 \mu \mathrm{L}$ of the $\mathrm{AChE}$ solution $\left(0.25\right.$ unit $\left.\mathrm{mL}^{-1}\right)$, from Electroporus electricus, in the same phosphate buffer were added. The assay solutions except substrate were preincubated with the enzyme for $30 \mathrm{~min}$ at room temperature. After preincubation, the substrate was added. The solution substrate consists of $\mathrm{Na}_{2} \mathrm{HPO}_{4}\left(40 \mathrm{mmol} \mathrm{L}^{-1}\right)$, acetylthiocholine $\left(0.24 \mathrm{mmol} \mathrm{L}^{-1}\right)$ and $5,5^{\prime}$ '-dithio-bis(2-nitrobenzoic acid) (0.2 mmol L-1, DTNB, Ellman's reagent). Absorbance of the yellow anion product due to the sponteneous hydrolysis of substrate was measured at $405 \mathrm{~nm}$ for $5 \mathrm{~min}$ on a Microtiter plate reader (Multiskan EX, Thermo, Finland). The AChE inhibition was determined for each compound. The enzyme activity was calculated as a percentage compared to a control using only the buffer and enzyme solution. The compounds were assayed in the dilution interval of $500-15 \mu \mathrm{g} \mathrm{mL} L^{-1}$, and the alkaloid galanthamine was used as the reference compound. Each assay was run in triplicate and each reaction was repeated at least three independent times. The $\mathrm{IC}_{50}$ values were calculated by means of regression analysis.

\section{Acknowledgments}

Financial support from CONICET (PIP 5011), ANPCyT (PICT 13922) and UBA (X058) are gratefully acknowledged. C.M.S. is a research fellow of the National Research Council of Argentina (CONICET) and N.B.D. is a Research Member of the CONICET. Luis Astudillo Saavedra acknowledges Fondecyt Project 1050985 and Anillo PBCT ACT-38. Margarita Gutierrez Cabrera acknowledges Project PBCT (PSD-16) for a postdoctoral grant.

\section{References}

1. Lee, S-S.; Venkatesham, U.; Prasad Rao, C.; Lam, S-H.; Lin, J-H.; Bioorg. Med. Chem. 2007, 15, 1034.

2. Francis, P. T.; Palmer, A. M.; Snape, M.; Wilcock, G. K.; J. Neurol. Neurosurg. Psychiatry 1999, 66, 137.

3. Schliebs, R.; Arendt, T.; J. Neural Transm. 2006, 113, 1625.

4. Rösler, M.; Anand, R.; Cicin-Sain, A.; Gauthier, S.; Agid, Y.; Dal-Bianco, P.; Stähelin, H. B.; Hartman, R.; Gharabawi, M.; Brit. Med. J. 1999, 318, 633.

5. Villalobos, A.; Blake, J. F.; Biggers, C. K.; Butler, T. W.; Chapin, D. S.; Chen, Y. L.; Ives, J. L.; Jones, S. B.; Liston, D. R.; Nagel, A. A.; Nason, D. M.; Nielsen, J. A.; Shalaby, I. A.; White, W. F.; J. Med. Chem. 1994, 37, 2721.

6. Carotti, A.; de Candia, M.; Catto, M.; Borisova, T. N.; Varlamov, A. V.; Méndez-Álvarez, E.; Soto-Otero, R.; Voskressensky, L. G.; Altomare, C.; Bioorg. Med. Chem. 2006, 14, 7205.

7. Marco, J. L.; de los Ríos, C.; García, A. G.; Villarroya, M.; Carreiras, M. C.; Martins, C.; Eleuterio, A.; Morreale, A.; Orozco, M.; Luque, F. J.; Bioorg. Med. Chem. 2004, 12, 2199.

8. Martins Alho, M. A.; Baggio, R.; Garland, M. T.; D’Accorso, N. B.; Varela, O.; Carbohydr. Res. 2002, 337, 1397.

9. a) Fascio, M. L.; D’Accorso, N. B.; Synth. Commun. 2001, 31, 1913. b) Fascio, M. L.; Montesano, V. J.; D’Accorso, N. B.; J. Carbohydr. Chem. 2000, 19, 393.

10. Fascio, M. L.; Montesano, V. J.; D’Accorso, N. B.; J. Heterocycl. Chem. 1998, 35, 103.

11. dos Anjos, J. V.; Sinou, D.; de Melo, S. J.; Srivastava, R. M.; Carbohydr. Res. 2007, 342, 2440.

12. Gutiérrez, M.; Theoduloz, C.; Rodríguez, J.; Lolas, M.; Schmeda-Hirschmann, G.; J. Agric. Food Chem. 2005, 53, 7701.

13. Schmid, C. R.; Bryant, J. D.; Org. Synth. 1995, 72, 6.

14. Hoffmann, R. W.; Eichler, G.; Endesfelder, A.; Liebigs Ann. Chem. 1983, 11, 2000. 